\title{
Quasi-thermal comptonization and GRBs
}

\author{
G. Ghisellini ${ }^{1}$ and A. Celotti ${ }^{2}$ \\ 1 Osservatorio Astronomico di Brera, via Bianchi 46, I-23807 Merate, Italy \\ e-mail: gabriele@merate.mi.astro.it \\ 2 S.I.S.S.A., via Beirut 2-4, I-34014, Italy \\ e-mail: celotti@sissa.it
}

Received December 18, 1998; accepted January 26, 1999

\begin{abstract}
Quasi-thermal Comptonization in internal shocks formed between relativistic shells can account for the high energy emission of gamma-ray bursts (GRB). This is the dominant cooling mechanism if the typical energy of the emitting particles is achieved either through the balance between heating and cooling or as a result of electron-positron $\left(\mathrm{e}^{ \pm}\right)$pair production. Both processes yield sub/mildly relativistic energies. In this case the synchrotron spectrum is self-absorbed, providing the seed photons for the Comptonization process, whose spectrum is flat $[F(\nu) \sim$ const], ending either in an exponential cutoff or a Wien peak.
\end{abstract}

Key words: gamma rays: bursts - radiation mechanisms: non-thermal, thermal — X-rays: general

\section{The "equipartition" scenario}

The main radiation mechanism assumed to give rise to both the burst event and the afterglow is synchrotron emission, by electrons of random Lorentz factor $\gamma \sim$ $m_{\mathrm{p}} / m_{\mathrm{e}}$, emitting in a region at a distance $R \sim 10^{13} \mathrm{~cm}$ from the origin (e.g. Rees \& Mészáros 1994). At these distances the typical scattering optical depth of the entire shell, of width $\Delta R^{\prime}$, is $\tau_{\mathrm{T}} \sim 1$, and the equipartition magnetic field is $B \sim 10^{5} \mathrm{G}$.

This picture implicitly requires that:

1) The acceleration of the electrons is impulsive (i.e. on timescales much shorter than the cooling timescales), in order for their final random energy to be controlled by the equipartition condition.

2) The inverse Compton power must be at most of the same order of the synchrotron one. This requires that only a small fraction of the electrons are, at any moment, relativistic, to avoid excessive inverse Compton emission: only a very small part of shell volume must be active.
3) There cannot be copious pair production: if the density of electrons is increased by $\mathrm{e}^{ \pm}$pairs, the mean energy per lepton is less than $m_{\mathrm{p}} / m_{\mathrm{e}}$.

We consider these constraints quite demanding. The emitting region cannot be very compact, not to have a significant amount of pair production and it must have a very narrow width, to avoid to emit too much by the inverse Compton process. A more detailed discussion of the ideas contained in this paper can be found in Ghisellini \& Celotti (1999).

\section{Quasi-thermal comptonization}

If the above conditions are not satisfied one can envisage an alternative scenario where the key role is instead played by the balance between the cooling and heating processes. This would be favored if the emitting region occupies the entire shell volume rather than the narrow region associated with a planar shock.

An immediate prediction is that the typical energy of the emitting electrons is mildly relativistic, and the main radiation process is quasi-thermal Comptonization. This also implies the existence of a characteristic observed frequency of a few $\mathrm{MeV}$, controlled by the feedback introduced by the effect of $\mathrm{e}^{ \pm}$pair production.

Let us assume that the heating process for a typical electron lasts for the duration of the shell-shell interaction, $\sim \Delta R^{\prime} / c$. The maximum amount of energy given to a single lepton, $\sim m_{\mathrm{p}} c^{2} n_{\mathrm{p}}^{\prime} / n_{\mathrm{e}}^{\prime}$, corresponds to a total average heating rate $\dot{E}_{\text {heat }}=n_{\mathrm{p}}^{\prime} m_{\mathrm{p}} c^{3} / \Delta R^{\prime}$. The characteristic electron energy is given by balancing $\dot{E}_{\text {heat }}$ and $\dot{E}_{\text {cool }}$, i.e.: $\gamma^{2} \beta^{2} \approx \frac{n_{\mathrm{p}}^{\prime} m_{\mathrm{p}}}{n_{\mathrm{e}}^{\prime} m_{\mathrm{e}}} \frac{1}{1+U_{\mathrm{B}} / U_{\mathrm{r}}^{\prime}} \frac{3 \pi}{\ell^{\prime}}$,

where $n_{\mathrm{e}}^{\prime}$ includes a possible contribution from $\mathrm{e}^{ \pm}$pairs. A typical value for the compactness in this case is $\ell^{\prime}=270$ $\left(L_{46}^{\prime} / R_{13}\right)\left(\Delta R_{11}^{\prime} / R_{13}\right)$, and therefore $\mathrm{e}^{ \pm}$are at most moderately relativistic. Even though the particle distribution 
may not have time to thermalize, it will be characterized by this mean energy, which can be expressed as an "effective temperature" $\Theta \equiv k T /\left(m_{\mathrm{e}} c^{2}\right)$.

The small energy of the emitting particles implies:

1) the synchrotron emission is self-absorbed. It peaks at a comoving frequency $\nu_{\mathrm{T}}^{\prime} \sim 10^{14} \mathrm{~Hz}$.

2) the main radiation mechanism is multiple Compton scattering and the self-absorbed synchrotron emission is the source of soft seed photons.

If one defines a generalized Comptonization parameter $y \equiv 4 \tau_{\mathrm{T}} \Theta\left(1+\tau_{\mathrm{T}}\right)(1+4 \Theta)$, the ratio of the Compton to the synchrotron powers can then be approximated by $\mathrm{e}^{y}$, and thus in order to emit a Compton comoving luminosity $L_{\mathrm{c}}^{\prime}=10^{46} L_{\mathrm{c}, 46}^{\prime}$ erg s${ }^{-1}, y$ must be $y \sim \ln \left(L_{\mathrm{c}} / L_{\mathrm{s}}\right) \sim$ $11.5 \ln \left(L_{\mathrm{c}, 46}^{\prime} / L_{\mathrm{s}, 41}^{\prime}\right)$. With this value of $y$, and $\tau_{\mathrm{T}} \sim 1$, the Comptonized high energy spectrum has a $F(\nu) \propto \nu^{0}$ shape, while the relatively modest optical depth prevents a strong Wien peak to form. Therefore, very schematically, the resulting observed spectrum would extend between $\nu_{\mathrm{T}}^{\prime} \Gamma$ and $\sim 2 \Gamma k T / h$, with $F(\nu) \sim$ const.

The spectrum of a single shell will rapidly evolve: after the observed acceleration time, particles cool on a similar timescale, while the Comptonization spectrum steepens and the power decreases. In the time integrated emission any Wien hump and/or feature in the spectrum of individual shells will be smoothed out. The power-law continuum, if typical of all shells, would instead be preserved.

The $\mathrm{e}^{ \pm}$pair production process can play a crucial role. This would both increase the optical depth and act as a thermostat, by maintaining the temperature in a narrow range. Additional pairs will be also produced outside the shell region, increasing the lepton content of the surrounding medium. The maximum equilibrium temperature in steady sources in pair equilibrium with $\ell^{\prime}$ between 10 and $10^{3}$ is of the order $30-300 \mathrm{keV}$ (Svensson 1984). Note that additional pairs will be also produced outside the shell region, increasing the lepton content of the surrounding medium.

We conclude that an "effective" temperature $k T \sim$ $50 \mathrm{keV}$ and $\tau_{\mathrm{T}} \sim 4$ dominated by pairs, can be a consistent solution giving $y \sim 11$.

\section{Some consequences}

- If the high energy spectrum is due to quasi-thermal Comptonization, it will be sensitive to the amount of seed photons, which is bound to increase for successive shells. This will in turn increase the cooling, lower the particle temperature and thus produce a softer spectrum. This can qualitatively explain the hard-to-soft behavior of GRB emission and the (weak) correlation duration vs. hardness.

- If the intrinsic effective temperature is of the order of $50 \mathrm{keV}$, the observed Comptonized spectrum extends to $\sim 10 \Theta_{-1} \Gamma_{2} /(1+z) \mathrm{MeV}$ at the beginning of the burst. One would also expect that during the initial phases a
Wien peak at the electron temperature would be formed for (plausibly powerful) bursts with $\tau_{\mathrm{T}} \geq 3-5$.

- If the progenitors of GRBs are hypernovae, the density in the vicinity of the central source is dominated by the pre-hypernova wind. This can lead to optical depths around unity at $R \sim 10^{12}-10^{13}$, where shell-shell collisions are assumed to take place. There is then the possibility that the GRB events are due to shocks with this material. Therefore: i) in the case of shocks between shells and the pre-hypernova wind the large densities involved suggest that inverse Compton emission is favored with respect to the synchrotron process; ii) if the (still unshocked) interstellar material has total $\tau_{\mathrm{T}} \geq 1$, photons will be down-scattered, introducing a break in the emergent spectrum at the observed energy $511 /\left[\tau_{\mathrm{T}}^{2}(1+z)\right] \mathrm{keV}$. Furthermore, the interstellar matter will act as a "mirror", sending back the scattered photons, thus increasing the amount of Compton cooling.

\section{Conclusions}

In the equipartition synchrotron scenario, the equality between the energy density of protons, leptons and magnetic field leads to a remarkable good agreement with the observed spectra. For this to be achieved the acceleration of electrons has to be impulsive, take place in a very limited volume of the interacting shell, and the $\mathrm{e}^{ \pm}$density has to be small enough, not to lower the mean lepton energy.

At the other extreme, when the particle acceleration occupies the entire shell volume and/or lasts for a shell light crossing time, the mean lepton energy is controlled by the balance between the heating and the cooling rate. This leads to mildly or sub relativistic lepton energies: the synchrotron emission is inhibited by self-absorption, and provides soft photons for the dominant inverse Compton scattering. The ratio of the observed multiple Compton scattering and the self-absorbed synchrotron luminosities is $\sim 10^{5}$, and determines the required Comptonization $y$ parameter. As long as the compactness of the emission region is large, relativistic temperatures cannot be achieved, because in this case $\mathrm{e}^{ \pm}$are copiously produced, increasing the optical depth and decreasing the temperature. If, on the other hand, the temperature is low and the optical depth is large, photons are trapped inside the shell, and part of the luminosity is used to expand it. As a result, the observed radiation is formed when the system adjusts to have $\tau_{\mathrm{T}} \sim$ a few and $k T \sim 50 \mathrm{keV}$. This may be why GRBs are observed to emit $\mathrm{MeV}$ photons.

\section{References}

Ghisellini G., Celotti A., 1999, ApJL (in press)

Rees M.J., Mészáros P., 1994, ApJ 430, L93

Svensson R., 1984, MNRAS 209, 175 